\title{
Bilateral Pulmonary Emboli on Dabigatran
}

\author{
Emily Clark 1, 2 , Joshua Walker 1, 2 , Ilya Aleksandrovskiy 1, 2 , Latha Ganti 3, 2, 1, 4 \\ 1. Emergency Medicine, Ocala Regional Medical Center, Ocala, USA 2. Emergency Medicine, University of Central \\ Florida College of Medicine, Orlando, USA 3. Emergency Medicine, Envision Physician Services, Plantation, USA 4. \\ Emergency Medicine, HCA Healthcare Graduate Medical Education Consortium Emergency Medicine Residency \\ Program of Greater Orlando, Orlando, USA
}

Corresponding author: Latha Ganti, latha.ganti@ucf.edu

\begin{abstract}
We present the case of a 44-year-old female who presented to the emergency department (ED) via emergency medical services with a chief concern of shortness of breath and was found to have bilateral pulmonary emboli (PE) while taking the direct oral anticoagulant dabigatran. The authors highlight the importance of considering PE even in patients who are on anticoagulation.
\end{abstract}

Categories: Emergency Medicine, Hematology

Keywords: dabigatran, acute pulmonary embolism

\section{Introduction}

Pulmonary embolism (PE) is the third most common cause of death worldwide after stroke and heart attack [1]. Although the exact prevalence is unknown, the incidence of venous thromboembolism (VTE) has been estimated to be around 300,000-600,000 cases annually in the United States [2]. In parallel, longitudinal studies have shown rising annual PE incidence rates over time [3]. Untreated PE can have a mortality of up to $25 \%$ which decreases to $1 \%-5 \%$ when the patient is placed on anticoagulation [4]. PE most often occurs when a lower extremity deep vein thrombosis (DVT) travels to the lungs.

Many choices exist for anticoagulation. Since their development and in the general population, direct oral anticoagulants (DOACs) have become the preferred agent over vitamin $\mathrm{K}$ antagonists. Patients can be characterized into low-intermediate risk and high-intermediate risk based on clinical data [5]. Lowintermediate risk PE causes a right ventricular (RV) strain while high-intermediate PE causes both RV strain as well as elevated cardiac biomarkers. The treatment of submassive PE remains controversial. For patients with recurrent PE, subtherapeutic anticoagulation is the most common cause. In this case report, we describe the management of a patient who developed recurrent bilateral PE on dabigatran and the considerations for her ED management and inpatient treatment.

Review began 05/12/2021 Review ended 06/17/2021 Published 06/18/2021

\section{(๑) Copyright 2021}

Clark et al. This is an open access article distributed under the terms of the Creative Commons Attribution License CC-BY 4.0., which permits unrestricted use, distribution, and reproduction in any medium, provided the original author and source are credited.

\section{Case Presentation}

A 44-year-old female with past medical history significant for anxiety, seizure disorder, hypothyroidism, and multiple provoked and unprovoked DVTs and PE for which she took $75 \mathrm{mg}$ oral dabigatran twice daily presented to the ED with fatigue and shortness of breath. According to the patient, she was unable to walk from her bed to the bathroom without feeling winded. She also had sharp chest pain whenever she took a deep breath with pain radiating to the right side of her neck. She presented with similar symptoms and was admitted to the hospital two days prior and was diagnosed with a DVT of the left lower extremity and PE of the right pulmonary artery. She was discharged home after one night in stable condition with a prescription for $150 \mathrm{mg}$ twice daily dabigatran, which was double her previous dosage.

She had been taking her dabigatran for the last five years; however, over the past two months, she had multiple interruptions in taking it as she was instructed to stop taking dabigatran four days prior to and following a recent spinal surgery that was rescheduled multiple times. She also occasionally uses marijuana, smokes half a pack of cigarettes most days for the past 10 years, and drinks alcohol a couple of times a month. She denied oral contraceptive use.

A complete review of systems was performed and was significant for left inner thigh pain that was slowly worsening in the last few days. Vital signs obtained were oxygen saturation of $99 \%$ on room air, blood pressure $142 / 95 \mathrm{mmHg}$, temperature $37.2^{\circ} \mathrm{C}$, pulse 98 beats per minute, and respiratory rate 18 breaths per minute. She often required deep breaths between words when talking. She was not in acute distress, however. Her physical exam was significant for tachycardia, tachypnea, and tenderness to palpation over the left thigh with no obvious deformities, swelling, or rashes seen.

Differential diagnosis included but was not limited to PE, acute coronary syndrome, pneumothorax, aortic dissection, pericarditis, pneumonia, pleurisy, and gastro-esophageal reflux. The initial diagnostic workup was remarkable for leukocytosis and elevated troponin (Table 1). A d-dimer was not obtained as the patient's 


\section{Cureus}

pre-test probability of having a PE was too high, meaning we would have obtained a computed tomography angiogram (CTA) of the chest regardless.

\section{Chemistry}

POC Glucose (70-110)

Magnesium (1.8-2.5 mg/dL)

2

POC Troponin I (0.00-0.07 ng/mL)

$0.19 \mathrm{H}$

Troponin I (0.00-0.034 ng/mL)

$0.211 \mathrm{H}$

Sodium (136-145 mmol/L)

Potassium (3.5-5.1 mmol/L)

3.8

Chloride (98-107 mmol/L)

Carbon dioxide (22-34 mmol/L)

BUN (7-18 mg/dL)

Creatinine (0.6-1.3 mg/dL)

0.7

Est GFR (Non-Af Amer) $(>60 \mathrm{~mL} / \mathrm{min})$

BUN/Creatinine ratio (12.0-20.0 ratio)

Glucose (70-110 mg/dL)

Calcium (8.8-10.5 mg/dL)

9.1

Total bilirubin $(0.2-1.5 \mathrm{mg} / \mathrm{dL})$

Direct bilirubin (0.0-0.5 mg/dL)

AST (12-37 IU/L)

ALT (4-35 Unit/L)

Alkaline phosphatase (38-126 IU/L)

Total protein (6.4-8.2 g/dL)

Albumin (3.4-5.0 g/dL)

Coagulation

PT (9.4-12.5 s)

INR (0.8-1.1)

APTT (25.1-36.5 s)

Hematology

WBC (3.7-11.0 thou/mm3)

RBC (3.40-5.10 m/mcL)

$\mathrm{Hgb}$ (11.0-15.4 g/dL)

Hct (32.5-44.5\%)

MCV (82.5 -98.5 fL)

96.2

MCH (26.5-35.3 pg)

MCHC (32.8-35.7 g/dL)

PIt Count (150-440 thou/mm3) 


\section{Cureus}

MPV (fL)

Neut \% (Auto) (\%)

Lymph \% (Auto) (\%)

Mono \% (Auto) (\%)

4.2

Eos \% (Auto) (\%)

2.5

Baso \% (Auto) (\%)

Absolute Neuts (auto) (2.0-7.5 thou/mcL)

Absolute Lymphs (auto) (0.7-3.1 thou/mcL)

Absolute Monos (auto) (0.2-0.9 thou/mcL)

0.6

Absolute Eos (auto) (0.0-0.4 thou/mcL)

Absolute Basos (auto) (0.0-0.3 thou/mcL)

Immature Gran \% (\%)

Immature Gran \# (0.0-0.031 thou/mcL)

\section{TABLE 1: Patient's laboratory values.}

POC, point of care; GFR, glomerular filtration rate; BUN, blood urea nitrogen; AST, aspartate aminotransferase; ALT, alanine aminotransferase; PT, prothrombin time; APTT, activated partial thromboplastin time; WBC, white blood cell; RBC, red blood cell; Hgb, hemoglobin; Hct, hematocrit; MCV, mean corpuscular volume; $\mathrm{MCH}$, mean corpuscular hemoglobin; $\mathrm{MCHC}$, mean cell hemoglobin concentration; RDW, red cell distribution width; MPV, mean platelet volume

Computed tomography angiogram was significant for bilateral PE in the distal left main pulmonary artery extending into the left lower lobe pulmonary artery branches as well as distal right main pulmonary artery extending into the upper and lower lobes. The heart was at the upper limit of normal size (Figure 1).

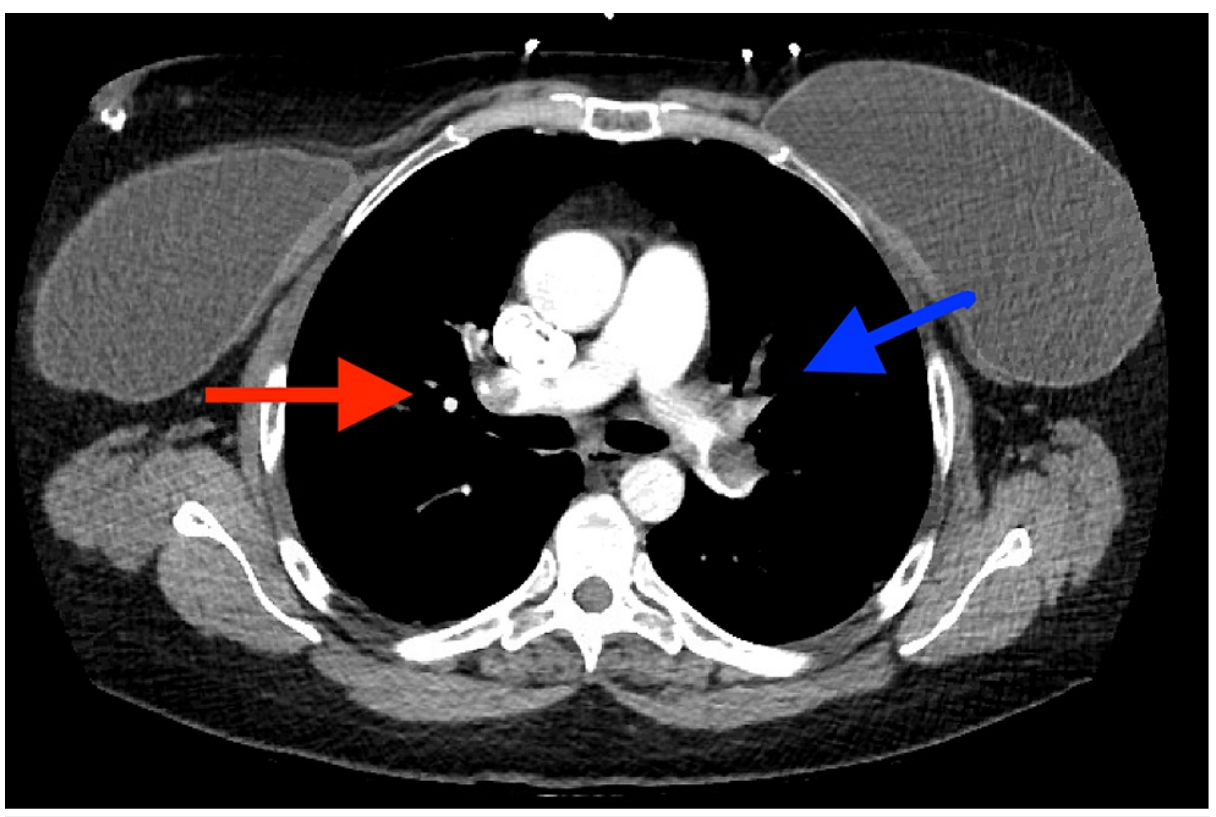

FIGURE 1: Pulmonary CTA demonstrating PE on the right (red arrow) and on the left (blue arrow).

CTA, computed tomography angiogram; PE, pulmonary emboli

An echocardiogram demonstrated preserved left ventricular function with an ejection fraction of $55 \%$ and was significant for a right ventricular systolic pressure of $57 \mathrm{mmHg}$ and left pleural effusion. Bilateral lower 


\section{Cureus}

extremity ultrasound re-demonstrated extensive left lower extremity DVT from left common femoral, superficial femoral, popliteal, and deep calf veins. The electrocardiogram demonstrated sinus tachycardia.

In consultation with hematology, a heparin drip was initiated. The patient was subsequently admitted to the medical floor. The patient was not a candidate for ekosonic endovascular system (EKOS) due to oral anticoagulation and showed an elevated international normalized ratio (INR). She was bridged to warfarin due to the patient's preference for no longer being on dabigatran. Hematology suggested that clot formation was likely secondary to medication non-compliance (intermittent medication use) rather than the failure of dabigatran. The patient's hospital course was complicated by multiple episodes of hematemesis as well as hematochezia that were addressed and resolved. She was ultimately bridged to warfarin and discharged home with oxygen as well as follow-up with hematology.

\section{Discussion}

Pulmonary emboli (thromboemboli in the lungs) most often occur when a lower extremity DVT travels to the lungs. Lower extremity DVTs often occur secondary to stasis, hypercoagulability, or local trauma (Figure 2).

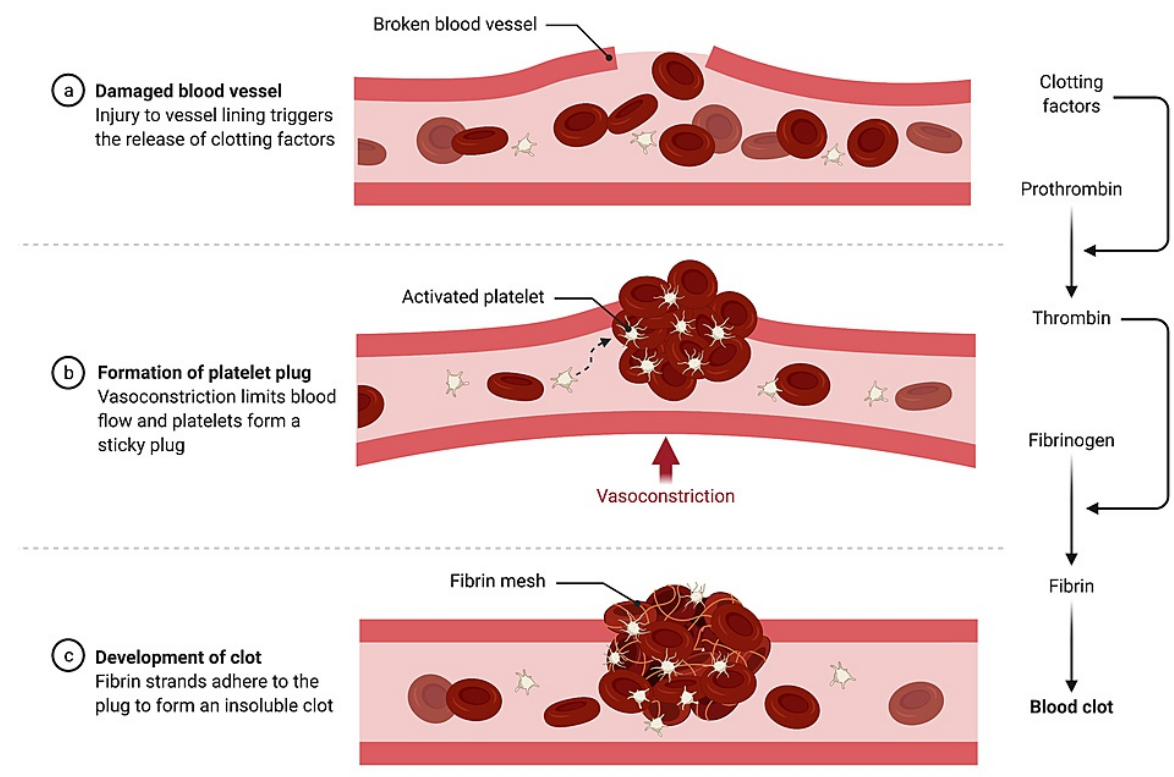

FIGURE 2: An illustration of the mechanism by which thromboemboli forms in a damaged blood vessel. Created with BioRender.com.

Risk factors [6] for thromboemboli such as DVT and PE are listed in Figure 3. 


\section{Cureus}

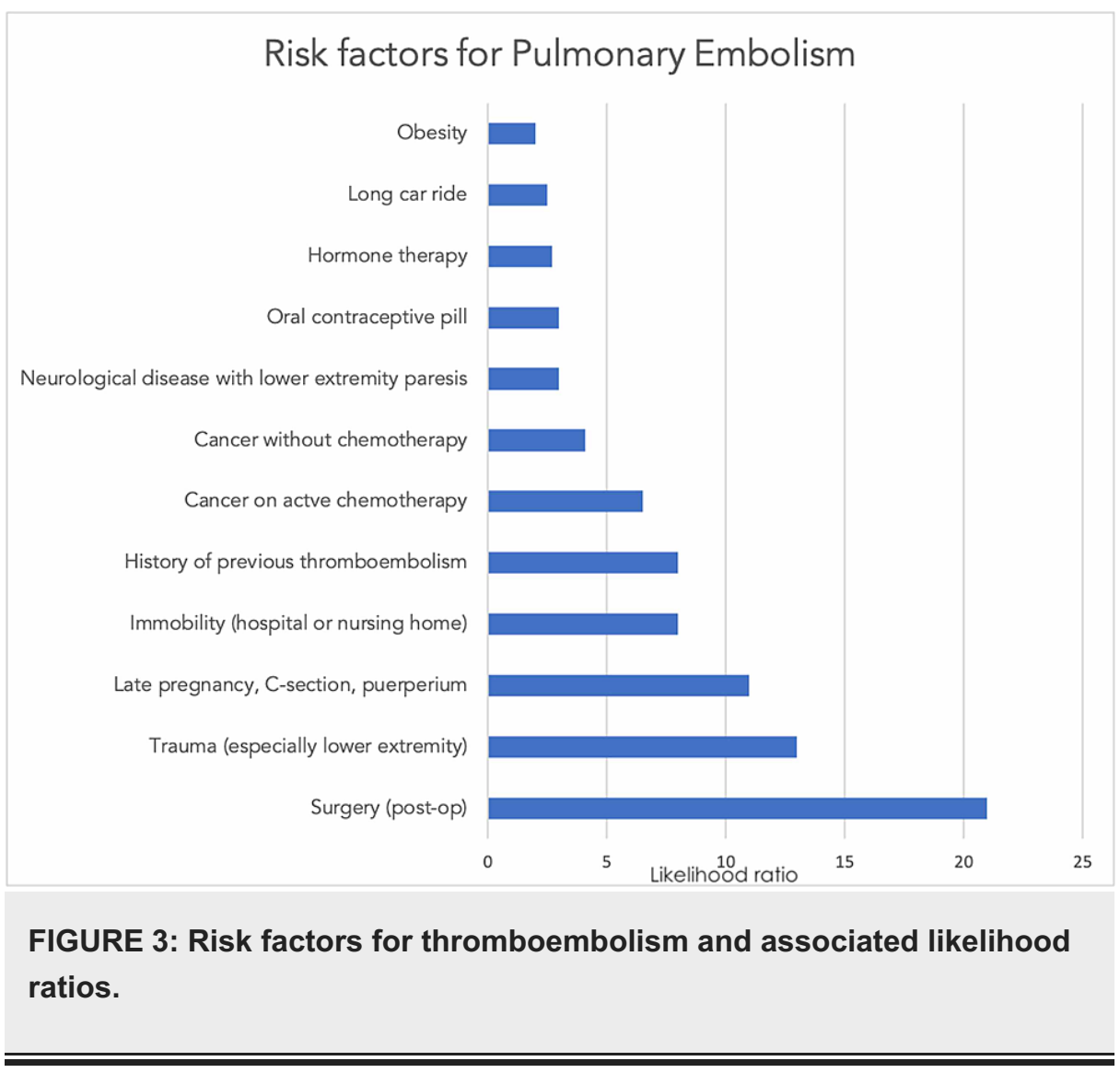

Dabigatran is a reversible, competitive direct thrombin inhibitor. It is able to bind and inhibit both free and clot-bound thrombin. Peak plasma concentrations of dabigatran occur within two hours of ingestion [7]. After the peak is reached, levels fall in a biphasic manner and result in a more than $70 \%$ decrease within four to six hours of ingestion. Approximately $35 \%$ of dabigatran is bound to plasma proteins and has a volume distribution of 50-70 L. In patients with normal renal function, approximately $80 \%$ of an IV dabigatran dose is excreted in urine with an elimination half-life of 12-17 hours; thus, dosing is dependent on creatinine clearance [7].

The Randomized Evaluation of Long-Term Anticoagulation (RE-LY) trial, a randomized noninferiority trial compared two doses of dabigatran with warfarin in 18,113 patients with atrial fibrillation. The trial demonstrated that at a dose of $110 \mathrm{mg}$, rates of stroke and systemic embolization were similar to warfarin, with lower rates of major hemorrhage. At a dose of $150 \mathrm{mg}$, rates of stroke and systemic embolization were lower in comparison to warfarin, however, with similar rates of major hemorrhage [7].

Dabigatran was approved by the US Food and Drug Administration for the prevention of embolic stroke in patients with non-valvular atrial fibrillation. When it was approved in 2010, it was the first new oral anticoagulant to be approved in the United States in 50 years. It is an attractive alternative to warfarin for patients and providers because it does not necessitate frequent lab monitoring due to its stable hematologic response. Also, it does not undergo CYP 450 metabolism and has few drug-drug and drug-food interactions.

At this time, laboratory measurements to determine the therapeutic level of dabigatran are limited and often not available in the ED. For example, thromboplastin time (TT), which measures a conversion of fibrinogen to fibrin in a plasma sample is sensitive to dabigatran levels. A normal TT likely indicates subtherapeutic levels of dabigatran [8]. However, it is not commonly available in the ED. Similarly, ecarin clotting time (ECT), which measures time to the formation of the clot of plasma sample with the addition of ecarin, also demonstrates a linear response to dabigatran serum levels. More common coagulation labs such as INR, prothrombin time, and aPTT have not been shown to be sensitive markers of dabigatran levels [9].

\section{Conclusions}

Understanding dabigatran's mechanism of action, laboratory monitoring of therapeutic levels, and pharmacokinetics are important for emergency physicians. As in this case study, this patient presented with concerning clot burden after inconsistent dabigatran usage. It was necessary to understand how to test for appropriate therapeutic levels of dabigatran as well as timing from the last dosage of dabigatran to initiating heparin therapy. It is also important for physicians to recognize the impact of intermittently discontinuing anticoagulation. 


\section{Additional Information}

Disclosures

Human subjects: Consent was obtained or waived by all participants in this study. HCA Centralized Algorithms for Research Rules on IRB Exemptions (CARRIE)/ IRB manager issued approval 2020-842. Consent was obtained or waived by all participants in this study. HCA Centralized Algorithms for Research Rules on IRB Exemptions (CARRIE)/ IRB manager issued approval 2020-842. HCA Centralized Algorithms for Research Rules on IRB Exemptions (CARRIE)/ IRB manager issued approval [Study \# 2020-842] Based on the information provided and attested as true, the research plan described does not require IRB oversight. This is because the investigators are either a) not engaging in research with human subjects as defined by federal regulations; b) engaging in research with human subjects deemed excluded from IRB oversight per 45CFR46.102(l) OR c) engaging in research with sufficient human subject protections in the design to meet one or more IRB exemption criteria set forth in 45CFR46.104. . Conflicts of interest: In compliance with the ICMJE uniform disclosure form, all authors declare the following: Payment/services info: All authors have declared that no financial support was received from any organization for the submitted work. Financial relationships: All authors have declared that they have no financial relationships at present or within the previous three years with any organizations that might have an interest in the submitted work. Other relationships: All authors have declared that there are no other relationships or activities that could appear to have influenced the submitted work.

\section{Acknowledgements}

This research was supported (in whole or in part) by HCA Healthcare and/or an HCA Healthcare affiliated entity. The views expressed in this publication represent those of the author(s) and do not necessarily represent the official views of HCA Healthcare or any of its affiliated entities.

\section{References}

1. Essien EO, Rali P, Mathai SC: Pulmonary embolism. Med Clin North Am. 2019, 103:549-564. 10.1016/j.mcna.2018.12.013

2. Cohen AT, Agnelli G, Anderson FA, et al.: Venous thromboembolism (VTE) in Europe. The number of VTE events and associated morbidity and mortality. Thromb Haemost. 2007, 98:756-764.

3. Konstantinides SV: Trends in incidence versus case fatality rates of pulmonary embolism: good news or bad news?. Thromb Haemost. 2016, 115:233-235. 10.1160/TH15-10-0832

4. Barritt DW, Jordan SC: Anticoagulant drugs in the treatment of pulmonary embolism. A controlled trial . Lancet. 1960, 1:1309-1312. 10.1016/s0140-6736(60)92299-6

5. Konstantinides SV, Torbicki A, Agnelli G, et al.: 2014 ESC guidelines on the diagnosis and management of acute pulmonary embolism. Eur Heart J. 2014, 35:3069a-3069k. 10.1093/eurheartj/ehu283

6. Heit JA, Silverstein MD, Mohr DN, Petterson TM, O'Fallon WM, Melton LJ 3rd: Risk factors for deep vein thrombosis and pulmonary embolism: a population-based case-control study. Arch Intern Med. 2000, 160:809-815. 10.1001/archinte.160.6.809

7. Ganetsky M, Babu KM, Salhanick SD, Brown RS, Boyer EW: Dabigatran: review of pharmacology and management of bleeding complications of this novel oral anticoagulant. J Med Toxicol. 2011, 7:281-287. 10.1007/s13181-011-0178-y

8. Stangier J, Rathgen K, Stähle H, Gansser D, Roth W: The pharmacokinetics, pharmacodynamics and tolerability of dabigatran etexilate, a new oral direct thrombin inhibitor, in healthy male subjects. Br J Clin Pharmacol. 2007, 64:292-303. 10.1111/j.1365-2125.2007.02899.x

9. Cuker A, Siegal DM, Crowther MA, Garcia DA: Laboratory measurement of the anticoagulant activity of the non-vitamin K oral anticoagulants. J Am Coll Cardiol. 2014, 64:1128-1139. 10.1016/j.jacc.2014.05.065 